\title{
Dynamics of Tubulin and Calmodulin in the Mammalian Mitotic Spindle
}

\author{
J. RICHARD MCINTOSH, ${ }^{a}$ WILLIAM M. SAXTON, ${ }^{a}$ DEREK \\ L. STEMPLE, ${ }^{a}$ ROGER J. LESLIE, ${ }^{a}$ AND \\ MICHAEL J. WELSH ${ }^{b}$ \\ aDepartment of Molecular, Cellular, and Developmental Biology \\ University of Colorado \\ Boulder, Colorado 80309 \\ and \\ ${ }^{b}$ Department of Anatomy \\ University of Michigan \\ School of Medicine \\ Ann Arbor, Michigan 48109
}

Microtubules (MTs) have attracted interest and experimental attention in part because they are involved in numerous cellular processes and in part because they are intriguingly dynamic. Many studies with light and electron microscopes have shown that MTs can change their location and extent of polymerization, depending on the physiological state of the cell. Efforts to study this dynamism and the cellular factors that control it have, however, approached the problem either indirectly or with low time resolution. For example, one can look at the changes in the birefringent retardation of the mitotic spindle as a result of anaphase or of some experimental treatment. Such observations are, however, indirect assays of tubulin assembly, because birefringence depends on the orientation and bunching of spindle MTs in addition to the amount of polymer and of other birefringent components present. One can directly study the effects of a given cellular perturbation on MT assembly by fixation and subsequent immunofluorescence, but such investigations are necessarily of low time resolution. MT assembly can be studied quickly and directly in vitro, but the systems of buffers and the proteins used for chemical analysis may not be an accurate reflection of the state within the cell. To study the assembly of MTs in their natural context, one wants a way to look directly at the polymerization behavior of tubulin in cells.

Fluorescence microscopy of dye-tagged molecules, or fluorescent analogue cytochemistry, is a powerful way to follow the polymerization dynamics of specific proteins in vivo. ${ }^{1}$ The requirements of the method are well defined: 1) a fluorophore-conjugated protein that is indistinguishable from the native macromolecule by all available functional tests, 2) a way to introduce the labeled protein into appropriate cells, 3) sufficient fluorescence signal to permit localization of the labeled protein at adequate space resolution, and 4) a device for following the fluorophore that can quantify the observed fluroescence. This approach to the study of tubulin was first used by Keith, Feramisco, and Shelanski ${ }^{2}$ who recognized the suitability of dichlorotriazinyl aminofluorescein (DTAF) as a fluorophore to label tubulin. They showed that DTAF tubulin will form cytoplasmic fibers that resembled MTs in their distribution and response to colchicine. Wadsworth and Sloboda ${ }^{3}$ confirmed these findings in a study of DTAF tubulin microinjected into sea urchin eggs, noting particularly the speed with which the fluoresecent tubulin analogue would incorporate into spindle-like structures.

Our laboratory has been investigating tubulin dynamics of living cells in collabora- 
tion with E. D. Salmon of the University of North Carolina (see his paper, this volume, p. 580). We have obtained additional evidence that DTAF tubulin is a good analogue for native mammalian brain tubulin in vitro ${ }^{4}$ and have shown that when this protein analogue is injected into living cells, the fluorescent fiber arrays that form are essentially identical to the distribution of total tubulin polymer as seen in fixed cells by immunofluorescence. ${ }^{5}$ A quantitative analysis of both the rates of fluorescence incorporation and of fluorescence redistribution after photobleaching have confirmed the previous impression of highly dynamic tubulin behavior in the mitotic spindle, ${ }^{6}$ and have demonstrated a rather rapid turnover in the interphase MT arrays. Details of this work have been published elsewhere. ${ }^{4-7}$ In this contribution we summarize the most important of our findings about tubulin and discuss plausible interpretations of the results. The behavior of tubulin will be compared with that of a less-prevalent spindle protein, calmodulin. The similarities and differences between the behaviors of these two spindle components are informative with regard to some aspects of spindle structure and to potentially powerful ways to learn more about the organization and function of the mitotic machinery.

\section{BEHAVIOR OF DTAF-TUBLIN IN VITRO}

Our conditions for conjugation of DTAF to MTs and for the subsequent purification of polymerization-active tubulin give a dye/protein molar ratio of about one. ${ }^{4}$ One-dimensional sodium dodecyl sulfate (SDS) gel electrophoresis shows that there is little protein aggregation in the final material, and that 90 to $100 \%$ of the dye is covalently bound to protein (it runs with the protein, not at the dye-front). When labeled protein is microinjected into cells, there is no fluorescence in the interphase nucleus, although unconjugated fluorophore injected into cytoplasm rapidly stains chromatin. We infer that all the dye, including the small fraction that runs at the dye-front, is tightly bound to tubulin under physiological conditions. Two-dimensional gel electrophoresis shows that both alpha and beta tubulin are labeled, beta about twice as strongly as alpha. The fluorescent protein has a slightly more acidic isoelectric point than unlabeled material, consistent with the conjugation of DTAF to amine groups on the protein.

DTAF-tubulin assembles and disassembles in the presence of heat-stable brain mircotubule-associated proteins (MAPs) at rates indistinguishable from those of unlabeled tubulin plus MAPs at equivalent concentrations. ${ }^{4}$ Pure DTAF-tubulin plus MAPs form true MTs, as seen by electron microscopy of negatively stained material. It adds to the ends of sea urchin flagellar MTs with the bias in polymerization rates characteristic of unlabeled brain tubulin: faster at the distal end of the flagellum. Both labeled and unlabeled tubulin add to MTs initiated by centrosomes at their ends distal to the centrosome. In neither of the above experiments is there an observable tendency for the DTAF-tubulin to stain the walls of existing MTs, suggesting that the fluorescence of MTs formed in the presence of labeled tubulin is a result of DTAF-subunits incorporated into the MT wall, not simply bound to the polymer's surface.

\section{BEHAVIOR OF DTAF-TUBULIN IN VIVO}

DTAF-tubulin microinjected into living mammalian cells incorporates into fibrous arrays that strongly resemble the cell's MT complexes. ${ }^{5,6}$ Immunofluroescent pictures 

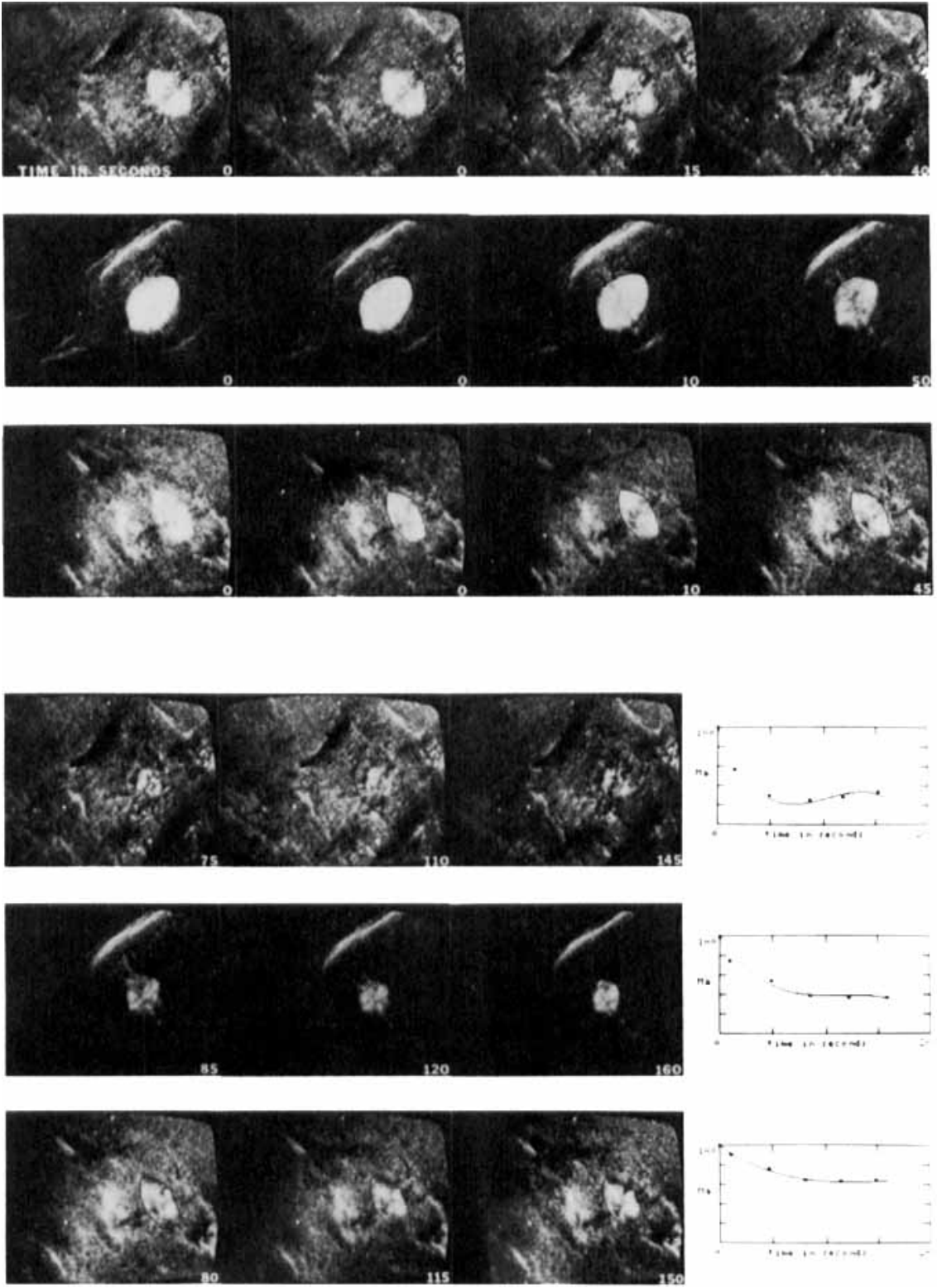

FIGURE 1. Cultured mammalian cells, strain $\mathrm{PtK}_{1}$, viewed with polarization optics. The images shown are photographs taken on $35 \mathrm{~mm}$ film from a video monitor displaying computer-averaged images of 16 successive video frames (about one-half sec of data). The top three rows of pictures are different cells, the first injected with $10 \mu M$ TC complex, the second with $10 \mu \mathrm{M}$ DTAF-TC complex, and the third with $10 \mu \mathrm{M}$ colchicine and bovine serum albumin at a mass concentration equal to the tubulin in the previous injections. The next three rows of pictures show the further development of birefringence with time. The points for the graphs shown at the end of each 
of cells fixed after equilibration with injected tubulin show that the image of rhodamine-labeled antitubulin, which stains all the MTs, and the fluorescein fluorescence of the injected tubulin are very similar. The D'TAF-tubulin image changes in expected ways upon treatment of the cells with nocodazole or taxol, and the time-dependent images of fluorescent tubulin over the cell cycle are consistent with the statement that the cell uses DTAF-tubulin as it does its own protein.

\section{CONTROLS FOR DTAF-TUBULIN INCORPORATION INTO MTS IN VIVO}

These observations do not exclude the possibility that the fluorescent tubulin analogue binds in vivo to the walls of existing MTs, staining their surfaces rather than incorporating into the walls of the cellular polymers. Whereas the evidence cited above from experiments in vitro makes this suggestion unlikely, it is not impossible. The ideal way to test this possibility would be to find a mutant cell in which all tubulin was missing under nonpermissive conditions, for example, an amber mutant in all relevant tubulin genes combined with a temperature sentitive supresser mutation. Unfortunately no such strain of injectable cells exists. We are therefore in the process of conducting several approximations to this experiment. One is based on the fact that tubulin forms a complex with colchicine that is slow to dissociate. ${ }^{8}$ One can form tubulin-colchicine (TC) complexes in vitro, separate them from unbound colchicine by gel filtration chromatography, and then inject them into cells to ask whether they will interfere with cellular physiology. Colchicine probably works to block MT assembly by forming a TC complex that adds to MT ends, thereby both distorting the normal lattice and reducing the rate of addition of further subunits. ${ }^{9,10}$ The blocking of MT assembly in vivo by injected TC complexes should therefore be an indication of the extent to which they compete with endogenous tubulin for end addition. By this logic, a comparison of the efficacy with which TC and DTAF-TC interfere with endogenous tubulin assembly is a measure of the capacity of DTAF-tubulin to incorporate into MTs in vivo.

We have made TC complexes and DTAF-TC complexes and injected them into mitotic mammalian cells. Either complex induces a rapid reduction in spindle birefringence (FIGURE 1). Spindle birefringence is slightly affected by injection of buffer containing bovine serum albumin, presumably a result of the perturbation caused by the injection itself. The birefringence is equivalently affected by injection of BSA plus $10 \mu M$ colchicine, suggesting that unbound colchicine leaves the cell faster than it can bind to the endogenous tubulin. Further, most of the cells that were injected with colchicine plus BSA entered an apparently normal anaphase, whereas only $10 \%$ of the cells injected with TC or DTAF-TC complex continued with mitosis. We conclude that the DTAF-TC is essentially as good at blocking the addition of endogenous tubulin as is the unlabeled TC complex. These data suggest that the cell does not distinguish between injected tubulin and DTAF-tubulin.

picture series were obtained by drawing a boundary around the spindle birefringence in the first image of each cell, then integrating the light intensity within and outside the boundary. The outside intensity was used to normalize all the pictures in one series to the same brightness level, then the inside intensity was used to estimate total spindle birefringence. The same boundary was aligned by eye on each image in the series to be sure that the same area was used to define the spindle in each case. The curves are cubics fit by a method of minimum sum squared error. The cell injected with colchicine and BSA entered anaphase, whereas the others did not. $\times 725$. 


\section{GEOMETRY OF INCORPORATION OF DTAF-TUBULIN IN VIVO}

One might hope that changes in the distribution of fluorescence during the incorporation of DTAF-tubulin might be informative about the cellular loci most active in tubulin assembly. So far, this hope has not been realized. The spindle seems to increase in fluorescence over its entire volume, and the fluorescence of the interphase MT array is buried in the fluorescence of unincorporated tubulin until the equilibration process is well advanced. We cannot say from the data in hand where subunits add to an MT in vivo.

\section{RATES OF INCORPORATION OF DTAF-TUBULIN MICROINJECTED INTO CELIS}

The behavior of DTAF-tubulin injected into interphase mammalian cells is rather what one might expect. The fluorescence is initially concentrated at the site of injection, then it spreads throughout the cytoplasm at a rate appropriate for diffusion. The cytoplasm is uniformly bright for several minutes, but over 3 to $20 \mathrm{~min}$, a fibrous fluorescence appears, consistent with the notion that DTAF-tubulin is slowly incorporating into cellular polymers. It is difficult to say when such a reaction is complete, but our best estimates of its rate suggest a half-time of $20 \pm 10 \mathrm{~min}(\mathrm{n}=11)$. In mitotic cells, the rate of incorporation is dramatically faster. The spindle becomes brighter than background before the newly injected protein has had time to diffuse across the cell. The mean half-time for fluorescence incorporation into 6 metaphase cells was $18 \pm 14 \mathrm{sec}$ and for 10 prometaphase asters was $14 \pm 7$ seconds.

Whereas these differences in rate are sufficiently marked to suggest an interesting difference between mitosis and interphase in the tubulin turn-over mechanisms, the injection experiments may be criticized on the grounds that the transient increase in tubulin concentration that follows injection may cause nonphysiological behavior. This criticism motivates a different approach to the problem in which there will be no concentration transient at the time of the experiment.

\section{RATES OF DTAF-TUBULIN TURNOVER AS SEEN BY FLUORESCENCE REDISTRIBUTION AFTER PHOTOBLEACHING}

We have used the method of fluorescence redistribution after photobleaching (FRAP) to circumvent the problems resulting from tubulin concentration transients immediately following injection. In these experiments cells are injected prior to study and allowed the time necessary for the injected protein to equilibrate with the endogenous tubulin pools. One then brings the preparation back to a microscope, equipped with a stage incubator to assure physiological temperatures, and finds previously injected cells by their fluorescence. A microbeam of laser light is imaged for a brief time $(0.5-0.05 \mathrm{sec})$ on a part of the fiuorescent cell, for example one spindle pole, and pictures are taken at successive times with a sensitive video camera to record the patterns and rates of fluorescence redistribution (FIgures 2 and 3). In such experiments there is no change in tubulin concentration at the time of the experiment, only a change in the visibility of some of the tubulin (bleached versus unbleached). The reappearance of fluorescence is due entirely to the motion of unbleached fluorophores, because the bleaching reaction is irreversible. With this approach one eliminates some of the concerns relevant to injection-incorporation experiments, but one introduces a new set of problems that requires a new set of controls, as described below. 

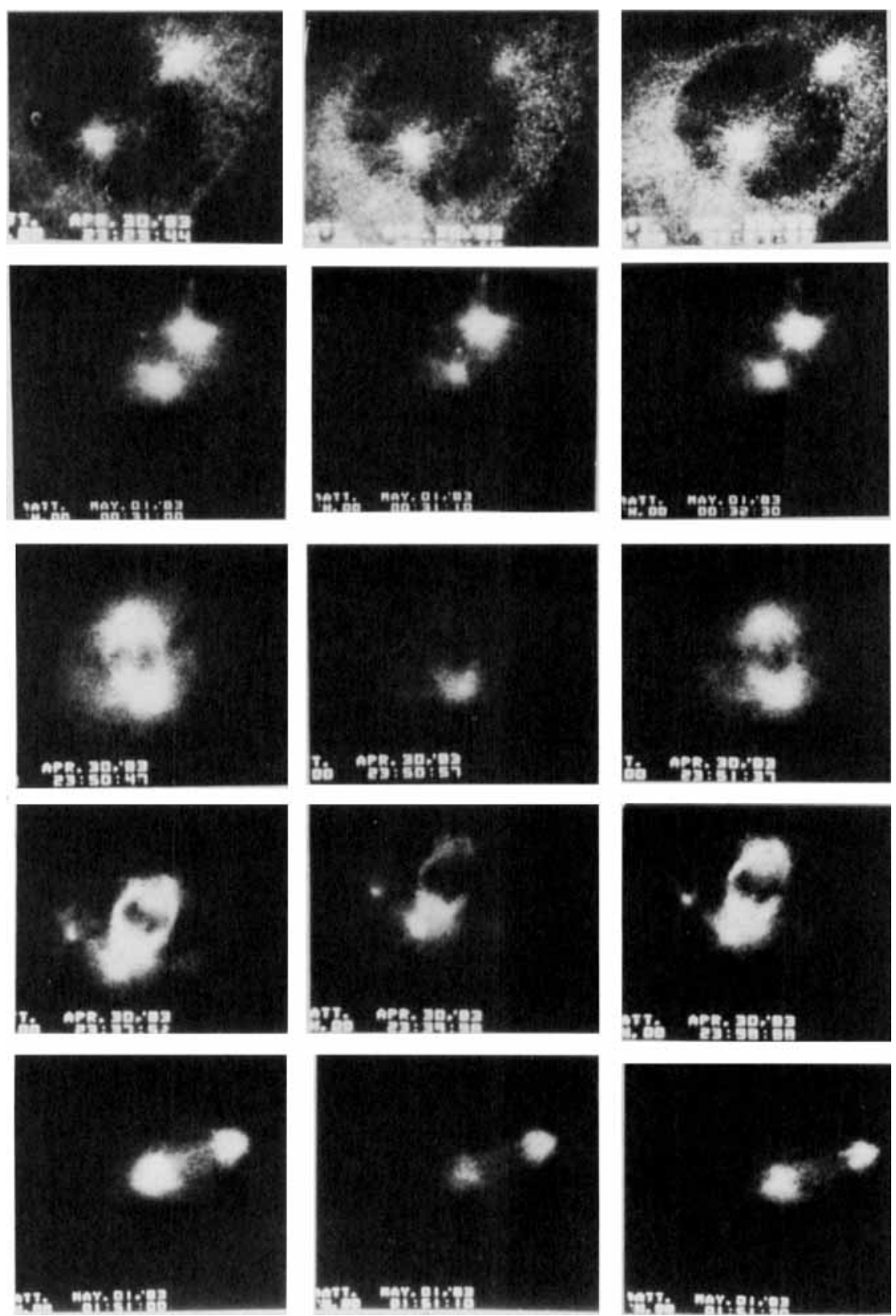

FIGURE 2. Mammalian cells injected with DTAF-tubulin at least one-half hour before viewing to permit equilibration of the fluorescent analogue with the cellular tubulin pool. Each cell is shown before, immediately after, then about a minute after photobleaching by a microbeam of argon laser light at $488 \mathrm{~nm}$. The rows of pictures are prophase, prometaphase, metaphase, early anaphase, and mid-to-late anaphase. Different extents of bleaching and various rates of recovery are shown, but in every case, the fluorescence returns. $\times 1200$. 

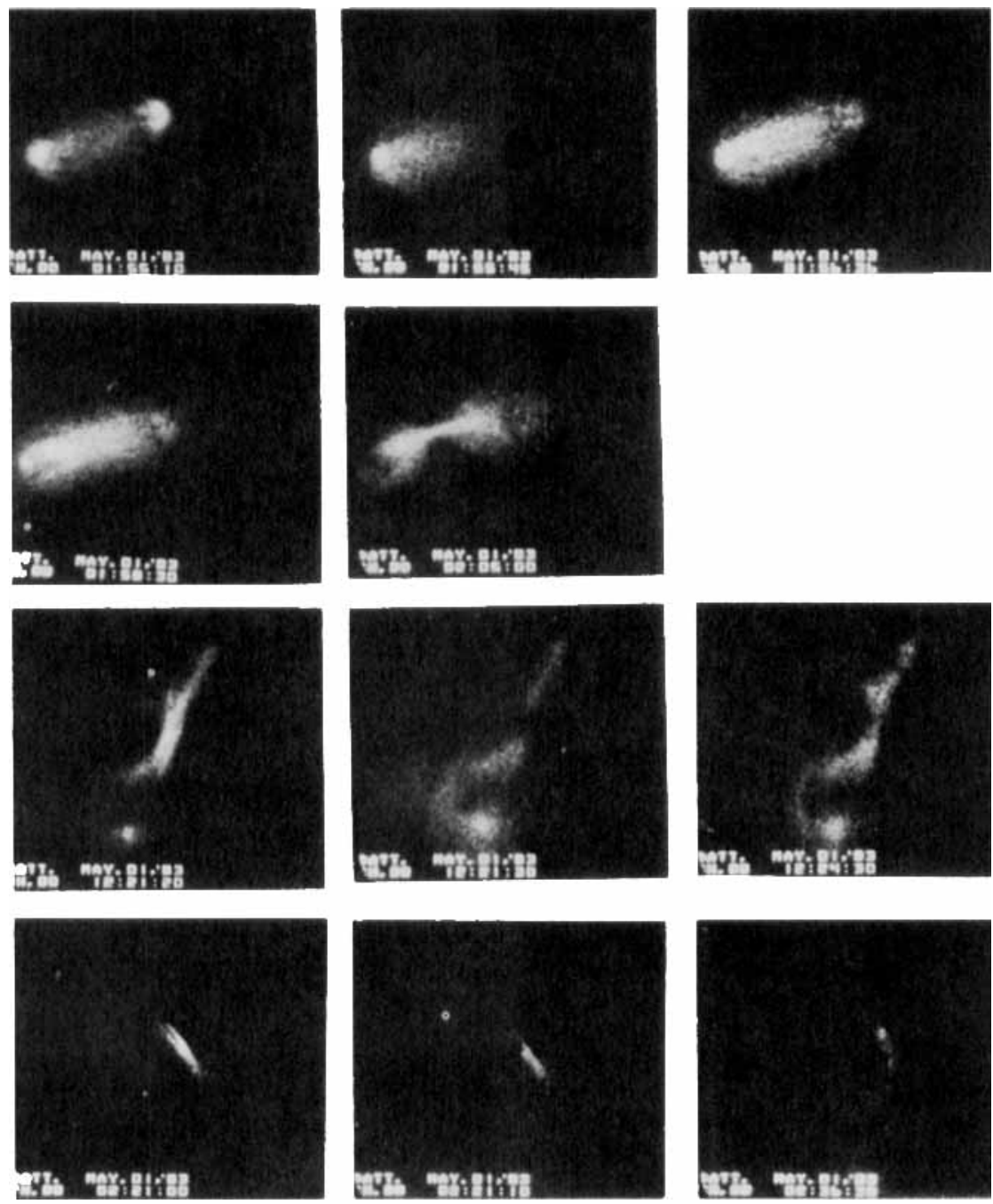

FIGURE 3. A set of images similar to that shown in Frgure 2. The anaphase of the first five images is the same as that shown at the end of FIGURE 2. These pictures demonstrate that a cell bleached twice will complete anaphase and cytokinesis. The different times of recovery suggest that the rate of fluorescence redistribution becomes slower in later anaphase. The images in the next two rows are of telophase midbodies and show that the trend to slower fluorescence redistribution continues into late mitosis. Midbody fluorescence redistribution is about as slow as that of interphase. $\times 1200$. 
We have measured the rates of FRAP of DTAF-tubulin in spindles and in the interphase MT complex of both PtK and BSC cells. The mean half-time for FRAP of metaphase spindles (indistinguishable between the two cell types) is $11 \pm 6 \mathrm{sec}$ $(n=19)$. For prometaphase asters, the half-time is $14 \pm 10 \mathrm{sec}(n=10)$. For interphase cells, the mean half-time is $200 \pm 85$ seconds. Thus our studies with FRAP confirm the difference in rates of tubulin turnover between mitotic and interphase cells, suggesting that this is a real feature of tubulin metabolism. The reasons and mechanisms for the difference are yet to be determined.

\section{GEOMETRY OF DTAF-TUBULIN TURNOVER AS SEEN BY FRAP}

If there were a substantial movement of MTs during the time that a photobleach was recovering, one would expect to see a translation of the bleached spot during its recovery. We have looked in both mitotic and interphase cells, but find no evidence for such a movement. In the spindle, the fluorescence comes back uniformly into the region that has been bleached. ${ }^{\text {S }}$ Therefore cellular MTs do not show any evidence of treadmilling, that is, the steady-state addition of subunits to one end and removal from the other. ${ }^{11}$ Spindle fluorescence recovery is fast enough that it is not possible to exclude a slow treadmilling of MTs during metaphase, but one can say with confidence that treadmilling is not the mechanism by which the spindle rapidly recovers fluorescence. In interphase on the other hand, the bleached spot sometimes moves a little during recovery. Examination of many cases shows that the spot occasionally moves in toward the centrosome and sometimes moves away. Bidirectional movement is confusing to a model of cellular tubulin turnover based on treadmilling and suggests that things are going on which we do not fully understand. Our observations on spot movement will be published in detail elsewhere.

\section{CONTROLS FOR MT DISRUPTION BY FLUORESCENCE BLEACHING}

The mitotic rate of FRAP is so fast that one is concerned about the possibility of some sort of experimental artifact. A simple explanation would be that rapid FRAP is a repair artifact of laser-induced MT destruction. Several lines of evidence suggest, however, that the process of bleaching does not disrupt MTs. Polymer made in vitro from pure DTAF-tubulin and heat-stable MAPs may be bleached to $80 \%$ of its initial fluorescence without change in its length distribution, as seen by negative staining. ${ }^{4}$ M'Ts bleached in vitro to negligible fluorescence will continue to elongate by addition of DTAF-tubulin at their ends, but they do not incorporate fluorescence into their surfaces at a measureable rate. We infer that bleached MT ends retain their polymerization activity, but that no new sites for subunit addition are opened up along an MT wall by the bleaching process. Microtubule structure is not disrupted by bleaching in vivo as seen either with polarization optics, ${ }^{6}$ by immunofluorescence with antitubulin, or by electron microscopy. ${ }^{5}$ Microtubule function is not disrupted by bleaching in vivo, as seen both by the continuation of normal anaphase in cells bleached two or three times during chromosome movement (FIGURE 3) and by the persistence of particle saltation along originally fluorescent MTs that have been bleached during interphase. 


\section{A CHEMICAL STUDY OF TUBULIN PHOTOBLEACHING}

The polymerization activity of DTAF-tubulin in vitro seems to be much more sensitive than the structure of MTs to damage by photobleaching in vitro. By trial and error we have identified conditions in which bleaching causes no visible damage to tubulin's capacity to polymerize, but there are circumstances in which the fluorescent analogue loses its activity upon irradiation. When pure DTAF-tubulin at $1 \mathrm{mg} / \mathrm{ml}$ plus $20 \mu \mathrm{g} / \mathrm{ml}$ unlabeled MAPs in a conventional piperazinediethanesulfonic acid (PIPES) polymerization buffer is bleached in a $1 \mathrm{~cm}$ path-length cuvette, using the $488 \mathrm{~nm}$ line from a $2 \mathrm{~W}$ argon ion laser at full power (about $650 \mathrm{~mW}$ ), approximately $5 \mathrm{~min}$ is required to obtain a bleach to $80 \%$ fluorescence. This treatment kills the polymerization activity of the tubulin, regardless of whether the protein was in polymer or monomer form at the time of bleaching. Electrophoresis of the irradiated material shows that some of the tubulin has become cross-linked to MAPs and other tubulin molecules, suggesting that MAP inactivation might contribute to the loss of activity. Fresh MAPs added after bleaching do not, however, restore polymerization, and all assembly activity is lost when only $20 \%$ of the tubulin is bleached. These results suggest that some sort of mobile intermediate is formed during bleaching that can damage tubulin molecules lying at a considerable distance from the bleaching event. Sheetz and Koppel ${ }^{12}$ have shown that the bleaching of a fluorophore attached to the outside of a red blood cell membrane will induce cross-linking of proteins that reside on the inner surface of the membrane, also suggesting that the active species in the bleach-induced cross-linking reaction can diffuse before it acts.

Photobleaching is thought to involve the formation of free radicals, ${ }^{13}$ so we have experimented with several additives to try to reduce the loss of polymerization activity experienced during bleaching of DTAF tubulin. Free radical scavengers, such as ascorbic acid, reduce the amount of damage done by a given energy of irradiation, but they also reduce the amount of photobleaching. When a preparation of DTAF tubulin is made $4 \mathrm{mM}$ in ascorbate, about five times more energy is required to achieve equivalent bleaching. When this energy is delivered, however, the resulting $20 \%$ bleach again induced a complete loss of polymerization activity. Sulfhydryl-reducing agents, such as mercaptoethanol, on the other hand, decrease the rate of loss of activity without affecting the rate of photobleaching. The cellular reducing agent glutathione is even more affective in protecting polymerization activity. In $5 \mathrm{mM}$ glutathione, there is also a reduction in the extent of protein cross-linking, as seen by gel electrophoresis, suggesting that the formation of inter- and intra-molecular disulfide bonds may contribute to the mechanisms of tubulin inactivation and cross-linking.

There are suggestions in the literature that the amount of damage to proteins during photobleaching depends on the rate at which the bleaching energy is delivered to the fluorophore. ${ }^{12}$ We therefore constructed a capillary flow cell whose inner diameter was about the same as the cross-section of the laser beam in our apparatus for microirradiation. DTAF tubulin was passed through the tube just fast enough that the time any one fluorophore would be exposed to the beam was about $0.5 \mathrm{sec}$, the longest time of irradiation in our cellular bleaching experiments. Sufficient material was then passed though the tube to permit its subsequent analysis for cross-linking and loss of polymerization activity. When DTAF tubulin was bleached by about $20 \%$ during a 0.5 sec irradiation, the loss of polymerization activity was substantially less than that seen in a 5 min bleach by 20 percent. Fast bleaching by $20 \%$ in glutathione leaves most of the DTAF tubulin active. When pure DTAF tubulin is diluted with unlabeled tubulin by $1: 10$, about the dilution achieved in an injection experiment, the resulting mixture may be bleached fast in glutathione with no observable loss in polymerization activity. Because these are the conditions that best approximate the experiments on FRAP in 
vivo, we think it likely that photobleaching of some of the injected fluorescent analogue does not seriously perturb tubulin assembly in the cell. Our experiments do not yet tell us, however, whether an individual DTAF-tubulin molecule may be bleached without losing its polymerization activity.

The reduction in photo-induced damage associated with fast bleaching at high power densities is consistent with a model in which protein damage is a two-step process. Our working hypothesis is that when the fluorophore is bleached: a comparatively long-lived intermediate is created. The intermediate can diffuse, and when it encounters a protein, it promotes oxidation of protein sulfhydryls, causing both denaturation and cross-linking. We suggest that the intermediate is itself photo-labile, so if the light intensity is very high, there is a good probability that the intermediate will be destroyed before it has a chance to act on a protein to which it has diffused. Such a two-step model makes several predictions that we are in the process of testing.

\section{POSSIBLE MECHANISMS FOR RAPID, UNIFORM MT TURNOVER IN MITOSIS}

The rapid rates of both incorporation and FRAP of DTAF-tubulin in mitotic cells suggest that there is something special about spindle MTs as compared with those of interphase. One possibility is that the concentration of MT ends in the spindle is much larger than that found in interphase. There is no rigorous study of MT length distributions available for mammalian spindles, but the data available for Dictyostelium, $^{14}$ for the alga Ochromonas, ${ }^{15}$ and for the fungus Puccinia ${ }^{16}$ suggest that at metaphase there is an essentially uniform distribution of MT lengths. One would expect, therefore, that there are plus ends of MTs scattered approximately uniformly throughout the metaphase spindle. This allows one to understand the uniformity of fluorescence incorporation into spindles, even if subunit exchange is confined to MT ends. In principle, the end exchange of subunits could also account for the fast rate of MT turnover, but a quantitative analysis of the rates observed here, using known disassembly rate constants and a model permitting subunit exchange at ends only, shows that simple condensation polymerization is unlikely to account for fast mitotic FRAP. ${ }^{6}$ To fit the data, one must assume a mean MT length of less than a micrometer, and the structure data on spindle tubule length exclude this possibility. A model based on treadmilling would permit subunit exchange at the observed rates, but makes the strong prediction of bleach spot movement during recovery, a prediction that is not born out by experiment.

Inoue ${ }^{17}$ has suggested that MT subunits in solution could exchange with material in the polymer wall at sites other than the polymer ends. One can imagine an "endotubulase" that catalyses this exchange at different rates during different times of the cell cycle and fits all the data available. There is, however, compelling evidence that the major site of subunit addition to MTs in vitro is at the polymer ends. ${ }^{18,19}$ It would therefore be appealing to find a model to account for fast MT turnover that is based on the assumption of subunit exchange at polymer ends.

Such a model has recently been proposed by Mitchison and Kirschner. ${ }^{20.21}$ They have shown that MTs in vitro display a dynamic instability that leads some MTs to elongate slowly while others rapidly disappear. They interpret their observations in the light of data on the rates of addition and loss for tubulin subunits bound to either guanosine triphosphate (GTP) or guanosine diphosphate (GDP) ${ }^{22}$ In this model, MTs are stable and grow as long as their ends are occupied by tubulin with GTP bound. If GTP hydrolysis catches up with subunit addition, generating a GDP-tubulin end, then the fast off-rate for this molecular species causes the MT to deploymerize rapidly to 
nothing. For MTs grown from a centrosome, fiber disappearance unveils a polymer initiation site, so a new MT can start to grow. Thus, a labile but constant MT array, like a metaphase spindle, can be thought to exist in rapid turnover as a result of occasional, catastrophic disassembly of a few MTs and continuous replacement of lost polymer by slow MT regrowth.

The Mitchison and Kirschner model would account for our data, so long as one assumes that the disappearance and reappearance of different MTs occurs asynchronously. The recovery of a bleached spot would then result from the rapid, occasional disappearance of individual bleached MTs, followed by their replacement, one by one, with MTs containing unbleached tubulin. The model also allows one to understand the stability of MTs attached to chromosomes, because they would have their plus ends capped by binding to the kinetochores, thereby preventing their fast disassembly. The model predicts that kinetochore MTs will show slow FRAP, a possibility that we are currently testing. The model also allows one to understand how the spindle might concentrate proteins on the kinetochore fibers, because they would be the oldest MTs in the metaphase spindle. If a particular MAP, for example, a calmodulin binding MAP, was slow in binding to MTs, then it would become concentrated on the more stable spindle fibers, because the others would not stay around long enough for appreciable binding to occur.

The difference in MT lability between mitosis and interphase can be understood in this model by the differences in the number of MTs initiated by the centrosomes at each cell cycle stage. ${ }^{23}$ This single change would alter the mean number of GTPtubulin subunits at the end of each MT, and thus the probability that a particular MT would suffer a catastrophic disassembly. The idea is thus very appealing in the face of information currently available. There are in addition several specific predictions of the model, so we should be able soon to test its validity more directly.

\section{THE DYNAMICS OF SPINDLE CALMODULIN}

Of the few spindle proteins other than tubulin that have so far been identified, calmodulin $(\mathrm{CaM})$ is one the most interesting, because it is known to regulate so many biological processes. ${ }^{24} \mathrm{CaM}$ has been identified as a spindle protein by immunofluorescence ${ }^{25}$ and by fluorescent analogue cytochemistry. ${ }^{26}$ We have therefore undertaken to measure its rate of turnover in the mitotic spindle.

We have derivatized $\mathrm{CaM}$ with either tetramethyl rhodamine ${ }^{26}$ or DTAF. Both fluorochromes yields a $\mathrm{CaM}$ analogue that is active by the criteria of showing a calcium-dependent shift in electrophoretic mobility ${ }^{24}$ and of conferring a calciumdependence on the specific activity of brain phosphodiesterase. Both analogues incorporate into mitotic spindles within seconds of injection into a mitotic cell. Neither shows specific localization in interphase other than being excluded from the nucleus.

All of our quantitative work on the rate of $\mathrm{CaM}$ turnover in vivo has been done by measuring the FRAP of DTAF-CaM during metaphase and anaphase. With the data in hand ( 8 metaphase cells and 2 anaphase), we cannot distinguish between the two mitotic stages. At metaphase, DTAF-CaM is distributed in a spindle-like morphology with a marked concentration at the poles and a characteristic staining of the kinetochore spindle fibers ${ }^{25}$ (FIGURE 4). Following fluorescence bleaching, there is first a rapid recovery of background, presumably due to diffusion, then a slower rise in the specific fluorescence above background (FIGURE 4). We have used the same sort of methods to quantify the rate of CaM FRAP as those that were applied for tubulin. The mean half-time for $\mathrm{CaM}$ fluorescence redistribution is $42 \pm 32 \mathrm{sec}$, about three times slower than the rate for tubulin. Preliminary results suggest that this rate is a result of 
two separate processes: a comparatively rapid FRAP on the spindle fibers and a slower FRAP in the vicinity of the pole.

The difference between turnover rates of $\mathrm{CaM}$ and tubulin is at first surprising, given the low molecular weight of the former and the intuitive sense that CaM will simply be bound to one or more spindle proteins, whereas tubulin will be incorporated into an MT wall. There are numerous possible explanations for the difference, and it seems too soon to speculate on its significance. In current work we are trying to measure the FRAP rate of the kinetochore MTs to see whether these more stable fibers turnover at the same slower rate as the $\mathrm{CaM}$, and we are planning to measure $\mathrm{CaM}$
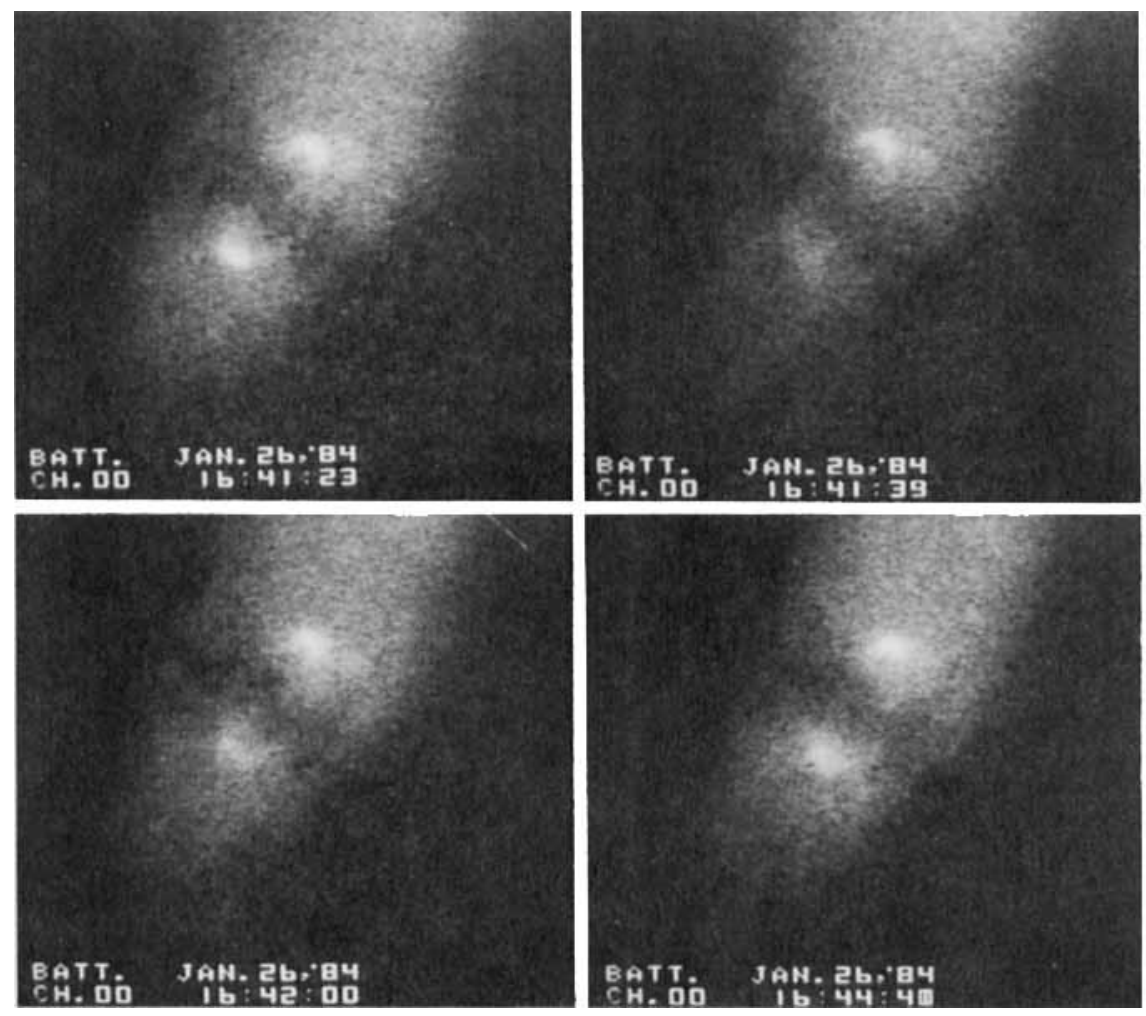

FIGURE 4. A PtK 1 cell injected with DTAF-calmodulin about $1 \mathrm{hr}$ before viewing. Images were prepared as in FIGURE 2. The bleaching and redistribution of fluorescence is clear. $\times 1700$.

FRAP with better time and space resolution to see if it confirms the heterogeneity in recovery, indicating the existence of several binding compartments.

\section{SIGNIFICANCE OF SPINDLE COMPONENT TURNOVER}

We hope that further work on the turnover of MTs will allow us to identify the significant pathways by which tubulin assembles and disassembles in cells. For 
example, if we can learn the site of subunit loss from the kinetochore MTs during anaphase, we will determine something quite important for specifying the location and mechanism of the spindle motors. Additional work on FRAP rates of other spindle components may help us to learn about their interactions with one another. It seems likely that interacting proteins will display similar turnover rates, and we may with FRAP analysis be able to group spindle proteins into classes that represent functional units.

The fact that $\mathrm{CaM}$ turns over more slowly than tubulin suggests that $\mathrm{CaM}$ does not bind directly to all the MTs of the half spindle. If the rate of CaM FRAP turns out to be slower even than that of the kinetochore MTs, then there will be a suggestion that at least some of the spindle CaM is bound not to MTs but to some other structural component of the spindle. Through a continuation of this kind of work, we hope to be able to learn a good deal about the interactions between different spindle components and to make progress toward understanding the mechanisms of mitosis.

\section{REFERENCES}

1. TAYlor, D. L., P. A. Amato, K. Luby-Phelps \& P McNeil. 1984. Fluorescence analogue cytochemistry. Trends Biochem. Sci. 9: 88-91.

2. KeIth, C. H., J. R. Feramisco \& M. ShelanSKI. 1981. Direct visualization of fluoresceinlabeled microtubules in vitro and in microinjected fibroblasts. J. Cell Biol. 88: 234-240.

3. WadSWORTH, P. \& R. D. SLOBODA. 1983. Microinjection of fluorescent tubulin into dividing sea urchin eggs. J. Cell Biol. 97: 1249-1254.

4. Leslie, R. J., W. M. Saxton, T. J. Mitchison, B. Neighbors, E. D. Salmon \& J. R. MCINTOSH. 1984. Assembly properties of fluorescent labeled tubulin in vitro before and after fluorescence bleaching. J. Cell Biol. 99: 2146-2156.

5. Saxton, W. M., D. L. Stample, R. J. Leslie, E. D. Salmon, M. Zavortink \& J. R. MCINTOSH. 1984. Tubulin dynamics in cultured mammalian cells. J. Cell Biol. 99: 21772186.

6. Salmon, E. D., R. J. Leslie, W. M. Saxton, M. L. Karow \& J. R. Mcintosh. 1984. Spindle microtubule dynamics in sea urchin embryos. J. Cell Biol. 99: 2164-2176.

7. Salmon, E. D., W. M. Saxton, R. J. Leslie, M. L. Karow \& J. R. Mcintosh. 1984. Diffusion coefficient of fluorescein-labeled tubulin in the cytoplasm of embryonic cells of the sea urchin. J. Cell Biol. 99: 2157-2164.

8. WILSON L. \& J. BRYAN. 1974. Biochemical and pharmacological properties of microtubules. Adv. Cell Biol. 3:21-71.

9. MARGolis, R. L. \& L. WiLSON. 1977. Addition of colchicine-tubulin complex to microtubule ends: the mechanism of substoicheometric poisoning. Proc. Natl. Acad. Sci. USA 74: 3466-3470.

10. SternLICHT, H. \& I. Ringel. 1979. Colchicine inhibition of microtubule assembly via copolymer formation. J. Biol. Chem. 254: 10540-10550.

11. Margolis, R. L. \& L. WiLSON. 1978. Opposite end assembly and disassembly of microtubules at steady state in vitro. Cell 13: 1-8.

12. SheETZ, M. P. \& D. E. Koppel. 1979. Membrane damage caused by irradiation of fluorescent concanavalin A. Proc. Natl. Acad. Sci. USA 76: 3314-3317.

13. Webr, W. W., L. S. Barak, D. W. TANK \& E- S WU. 1980. Molecular mobility on the cell surface. Biochem. Soc. Symp. 46:191 205.

14. McIntosh, J. R., U- P Roos, B. NeIGHBors \& K. L. MCDONald. 1985. Architecture of the microtubule component of mitotic spindles from Dictyostelium discoideum. J. Cell Sci. 75: 93-129.

15. Tippit, D. H., L. Pillus \& J. D. Pickett-Heaps. 1980. Organization of the spindle microtubules in Ochromonas dancia. J. Cell Biol. 87: 531-545.

16. Tippit, D. H., C. T. Fields, K. L. O'Donnell, J. D. Pickett-Heaps \& D. L. MclaughLIN. 1984. The organization of microtubules during anaphase and telophase spindle elongation in the rust fungus Puccinia. Eur. J. Cell Biol. 34: 34-44. 
17. INOUE, S. \& H. RitTeR. 1975. Dynamics of mitotic spindle organization and function. In Molecules and Cell Movement. S. Inoue \& R. E. Stephens, Eds.: 3-30. Raven Press. New York.

18. Bryan, J. 1976. A quantitative analysis of microtubule elongation. J. Cell Biol. 71: 749767.

19. BERGEN, L. G. \& G. G. BoRISY. 1980. Head-to-tail polymerization of microtubules in vitro. Electron microscopic analysis of seeded assembly. J. Cell Biol. 84:141-150.

20. MITCHISON, T. \& M. KIRSCHNER. 1984. Microtubule assembly nucleated by isolated centrosomes. Nature (London) 312: 232-237.

21. Mitchison, T. \& M. KIRSChNER, 1984. Dynamic instability of microtubule growth. Nature (London) 312: 237-241.

22. CARLIER, M-F., T. L. HILL \& Y. CHEN. 1984. Interference of GTP hydrolysis in the mechanism of microtubule assembly: an experimental study. Proc. Natl. Acad. Sci. USA 81: 771-775.

23. SNYDER, J. A. \& J. R. MCINTOSH. 1975. Initiation and growth of microtubules from mitotic centers in lysed cells. J. Cell Biol. 67: 744-760.

24. Means, A. R., J. S. Tash \& J. G. Chafouleas. 1982. Physiological indications of the presence, distribution, and regulation of calmodulin in eukaryotic cells. Physiol. Rev. 62: $1-39$.

25. Welsh, M. J., J. R. Dedman, B. R. Brinkley \& A. R. Means. 1979. Tubulin and calmodulin. Effects of microtubule and microfilament inhibitors on localization in the mitotic apparatus. J. Cell. Biol. 81: 641-655.

26. ZAVORTINK, M., M. J. WeLSH \& J. R. McINTOSH. 1983. The distribution of calmodulin in living cells. Exp. Cell. Res. 149: 375-385. 